\title{
Enfermedad por Reflujo Gastroesofágico
}

\author{
Gastroesophageal Reflux Disease
}

\author{
Carlos Manterola*
}

MANTEROLA, C. Enfermedad por reflujo gastroesofágico. Int. J. Med. Surg. Sci., 3(1):795-809, 2016.

RESUMEN: La enfermedad por reflujo gastroesofágico (ERGE) es una de las causas de consulta más frecuentes en atención primaria y en gastroenterología. Más el $40 \%$ de la población general experimenta pirosis y regurgitación ocasional debido a ERGE. Es una entidad nosológica compleja, y la fisiología y patogénesis aún no están totalmente aclaradas. Sin embargo, alteraciones a nivel del esfínter esofágico inferior, de la motilidad esofágica o del vaciamiento gástrico pueden producir o empeorar la ERGE. Hay diversos enfoques diagnósticos y terapéuticos; sin embargo, el uso de inhibidores de la bomba de protones a permanencia y la fundoplicatura (cirugía antirreflujo), son las opciones más efectivas.

PALABRAS CLAVE: Enfermedad por reflujo gastroesofágico; Gastroenterología; Complicaciones esofágicas; Endoscopia.

\section{INTRODUCCIÓN}

La enfermedad por reflujo gastroesofágico (ERGE) se ha de entender como una condición crónica, de carácter recurrente, que puede ser influenciada por diversos factores exógenos (algunos alimentos, tabaco, alcohol, etc.), y endógenos (obesidad, estrés, hernia de hiato, etc.); constituyéndose de esta forma en uno de las causas de consulta más frecuentes no sólo en el ámbito de la gastroenterología sino que también de la medicina general (American College of Gastroenterology, 2016; Armstrong et al., 2005; Asociación Española de Gastroenterología, 2016).

La ERGE se caracteriza por una fisiopatología compleja y multifactorial; y por un variado espectro sintomático en el cual predominan la pirosis y la regurgitación. Sin embargo, en ocasiones aparecen síntomas respiratorios o dolor torácico retroesternal (ACG; AEG)

Es frecuente el hallazgo de complicaciones esofágicas a la endoscopía, como erosio- nes, úlceras, estenosis y metaplasia de Barrett; hasta en el $60 \%$ de los pacientes sintomáticos (Armstrong et al.).

\section{REPORTE DE CASO}

Una paciente de 34 años, consulta por pirosis y regurgitación diaria de 12 meses de evolución, asociado a episodios de dolor torácico ocasional desde hace 4 meses, a lo que se agregó disfagia a sólidos en las últimas semanas. No refiere baja de peso ni hemorragia digestiva y se automedica con omeprazol de vez en cuando para el control de sus síntomas, los que disminuyen parcialmente y por algunas horas tras la ingestión del fármaco antes señalado. Refiere además que ha notado que el tono de su voz ha cambiado y que ocasionalmente presenta tos y carraspera matinal.

Concurre con una endoscopía reciente en la que se aprecia congestión y friabilidad de la

*Departamento de Cirugía. CEMyQ (Centro de Estudios Morfológicos y Quirúrgicos), Universidad de La Frontera, Temuco, Chile. 
mucosa esofágica distal y dos erosiones no confluentes a este nivel.

Objetivos de aprendizaje.

Conocer las manifestaciones clínicas de un paciente con enfermedad por reflujo gastroesofágico (ERGE).

- Reconocer las diversas pruebas diagnósticas que se pueden utilizar en estos casos, sus indicaciones y el objetivo de la petición de cada una de ellas.

Mencionar y explicar las complicaciones evolutivas de la ERGE.

- $\quad$ Familiarizarse con las medidas terapéuticas disponibles para un paciente con ERGE.

- Exponer las indicaciones quirúrgicas para un paciente con ERGE.

\section{DEFINICIÓN O CONCEPTO}

El reflujo gastroesofágico es un fenómeno fisiológico en la especie humana, sin embargo y por razones diversas; lo que se considera normal, en ocasiones se torna enfermedad, pues aparecen síntomas frecuentes (situación que, para quien la padece puede resultar invalidante), complicaciones de diversa índole e imposibilidad de tratamiento (Cohen et al., 2006).

Esta entidad, que se reconoce como ERGE, constituye un desafío clínico debido a su elevada frecuencia en la población, lo complejo de su fisiopatología y tratamiento (AEG; ACG).

\section{ASPECTOS EPIDEMIOLÓGICOS}

Como se señaló con antelación, la ERGE constituye una causa frecuente de consulta. Sus síntomas se consideran tan habituales, que se ha reportado una frecuencia de hasta $42 \%$ de éstos en población general adulta (ACG).

Por otro lado, se estima que hasta un 30 $\%$ de los sujetos sintomáticos tienen esofagitis y que un $70 \%$ requieren de tratamiento regular para el control de su sintomatología, lo que puede afectar notoriamente su calidad de vida (AEG; Armstrong et al.).

Existen pocos reportes acerca de la prevalencia de ERGE en población general, pues la mayor parte de ellos han sido realizados en po- blaciones muy particulares (como adultos mayores, familiares o amigos de pacientes hospitalizados, e incluso en discapacitados mentales (Avida et al., 2001), embarazadas, pacientes en hemodiálisis, gemelos y deportistas) (Cameron et al., 2002; Collings et al., 2003); utilizando instrumentos sin evidencias de validación previa ni de su real capacidad de reproducibilidad en la medición; y aplicados a través de métodos controvertibles, como el teléfono y el correo.

En un estudio piloto realizado por nuestro grupo de trabajo, en el que se aplicó una escala válida y confiable a 364 sujetos provenientes de la población general urbana de una ciudad del sur de Chile, con un intervalo de confianza de $95 \%$, encontramos que la prevalencia de ERGE fue de $52,8 \%$. Al incrementar la muestra a 1069 sujetos encuestados, y estrechando el intervalo de confianza a $99 \%$, se determinó una prevalencia de ERGE de 44,8 \% (Manterola et al., 2005).

Ahora bien, si consentimos en que la ERGE es una enfermedad frecuente; $y$, que se asocia a un riesgo potencial de generar morbilidad asociada que implica costos sustanciales en hospitalizaciones y terapéuticas especializadas (se estima que la prevalencia de esofagitis por reflujo es probablemente de un $5 \%$ a $10 \%$ de los individuos que acuden a consultorios y policlínicas; que la estenosis secundaria puede pesquisarse en un $10 \%$ de los pacientes con síntomas; que la metaplasia de Barrett puede encontrarse entre un $4,5 \%$ y $20,0 \%$ de los pacientes sintomáticos, y que la incidencia de adenocarcinoma en pacientes con esófago de Barrett es de 1:52), se podría decir entonces, que representa un problema de la salud pública (ACG).

Por otra parte, siendo la ERGE una enfermedad no infecciosa, no presentar variaciones estacionales, ni estar sujeta a otras variables de tendencia secular, se supone que la frecuencia y consulta motivada por ella se mantiene relativamente estable dentro de un lapso de tiempo relativamente largo. Este hecho, permite que basado en estimaciones previas, los administradores sanitarios puedan tomar medidas sanitarias al menos en el mediano plazo (AEG; Armstrong et al.). 


\section{ELEMENTOS FISIOPATÓLOGICOS}

Es evidente que la ERGE constituye una entidad de suyo compleja analizada desde todo punto de vista en especial del de su fisiopatología. La ERGE, puede explicarse mediante la comprensión de un modelo multifactorial, donde diversos mecanismos, anatómicos y fisiológicos pueden presentar fallos de cuantía diversa.

De modo de generar un funcionalismo apropiado, el esófago requiere de la acción de zonas motoras, que. El esófago posee tres zonas motoras; el esfínter esofágico superior (EES), el cuerpo esofágico y el esfínter esofágico inferior (EEI); la adecuada función de cada una de ellas, permite la propulsión del bolo alimentario hacia distal e impide el ulterior reflujo de este material hacia el esófago (Fig. 1) (Manterola et al., 2001a, 1998).

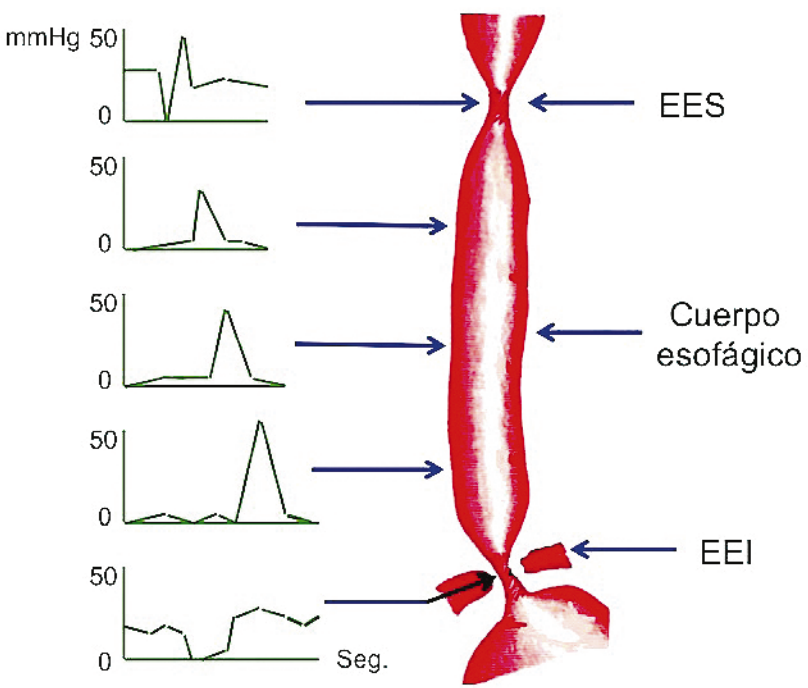

Fig. 1. Esquema de la fisiología del esófago y sus zonas motoras (EES, cuerpo esofágico y EEI). Se aprecia la respuesta deglutoria del EES, la motilidad del cuerpo esofágico y la respuesta deglutoria del EEI.

EI EES constituye un mecanismo esfinteriano de 2 a $4 \mathrm{~cm}$ de longitud, en el que se registra una presión de reposo de entre 45 y $90 \mathrm{mmHg}$. Su extremo superior está cerrado por un mecanismo hermético al paso del aire, el que se relaja durante la deglución, el vómito y el eructo. Esta estructura se forma en base a las fibras del músculo cricofaríngeo entrelazán- dose con la unión faringoesofágica (Manterola et al., 2001b, 1998).

El cuerpo esofágico es una estructura tubular, localizada entre el EES y EEI. La mayor parte del tiempo aparece como una cavidad virtual, con una presión intraluminal negativa de entre -1 y $-5 \mathrm{mmHg}$; la que se hace más negativa con la inspiración. Esta estructura es la que permite el paso del bolo alimentario al estómago; para lo cual cuenta con factores coadyuvantes como la fuerza de gravedad, la bipedestación, la salivación, la deglución y la propulsión esofágica o peristalsis (generada entre otras por el estímulo deglutorio); siendo este último el más importante y se genera por ondas o contracciones primarias, peristálticas o propulsivas las que progresan en sentido céfalo-caudal, con presiones mayores hacia distal. A estas ondas se les estudia su amplitud (registrada en $\mathrm{mmHg}$ ) y duración (registrada en segundos); registrándose además la frecuencia de aparición de ondas secundarias (no se relacionan con la deglución, y aparecen como respuesta a un estímulo local como un episodio de reflujo o remanentes del bolo alimentario que no fue propulsado por la onda primaria) y terciarias (no constituyen peristalsis, pero están presentes en baja frecuencia en los seres humanos; y son consideras ondas patológicas, pues son simultáneas, no-propulsivas, de baja amplitud y larga duración) (Manterola et al., 2001a, 2001b, 1998).

EI EEI, es una estructura especializada que se localiza en la unión entre el esófago y el estómago, y que mantiene la diferencia de presiones entre la cavidad torácica, y la cavidad abdominal. Tiene una longitud de 2 a $4 \mathrm{~cm}$, y una presión de reposo que fluctúa entre 12 y $25 \mathrm{mmHg}$. Está compuesto de músculo liso y constituido por la muscular de la mucosa, fibras circulares y longitudinales (Manterola et al., 2000, 2001a, 2001b).

En este modelo multifactorial que permite entender la ERGE, sus componentes se pueden agrupar esquemáticamente en 2 grupos: los componentes anatómicos y los fisiológicos; sin embargo, el que juega el rol más preponderante de todos ellos es el EEI, cuya alteración es fundamental en la patogenia de la ERGE. 
Entre los componentes anatómicos se menciona el EEI, la roseta mucosa, el ángulo de His y los pilares diafragmáticos; y el segmento intra abdominal del esófago. El EEI, es una zona de presión de 2 a $4 \mathrm{~cm}$ de longitud, que se localiza en la región distal del esófago y generalmente en relación con la línea $\mathrm{z}$, o zona de transición entre las mucosas esofágica y gástrica; sin embargo, su trascendencia no excluye la importancia de otros factores anatómicos, que actuarían por un lado, ejerciendo un efecto directo sobre el EEI favoreciendo su acción; y por otro, en forma indirecta aportando efectos adicionales de contención. Respecto de la roseta mucosa, se puede decir que son pliegues mucosos en el cardias que se pensaba actuaban como válvula, pero este mecanismo está en la actualidad descartado. En relación al ángulo de His y los pilares diafragmáticos, se puede mencionar que la desembocadura del esófago en el estómago es de tipo oblicua, de arriba abajo y de derecha a izquierda, lo que da forma a un ángulo abierto, denominado ángulo de His; área en la que el hiato diafragmático presenta dos fascículos musculares que abrazan al esófago, de forma tal que al contraerse con maniobras como la tos, la inspiración forzada, o el incremento súbito de la presión intra abdominal, desplazan al esófago hacia abajo y atrás, en dirección de su angulación, coadyuvando a la obliteración de su lumen. Finalmente, y respecto del segmento intra abdominal del esófago, se puede comentar que una vez que el esófago cruza el hiato, el esófago presenta un segmento de 2 a $4 \mathrm{~cm}$ de longitud, que se encuentra en la cavidad abdominal y que por ende es sometido a presiones positivas y mayores que las encontradas en el esófago torácico, razón por la cual, se cree que esta presión positiva contribuye a este segmento del esófago en mantener su luz obliterada, hecho que ayudaría a la prevención del reflujo; no obstante ello, para algunos investigadores, este mecanismo no sería relevante (Nocon et al., 2007; Manterola et al., 2000).

Entre los componentes fisiológicos se ha de tener en cuenta la función del EEI, el aclaramiento esofágico, el rol que pueden tener distintos tipos de reflujo, la composición del líquido refluido; y la respuesta de la mucosa esofágica. En relación al funcionalismo del EEI (tono basal, longitud y existencia de relajacio- nes espontáneas transitorias), es importante mencionar el rol del tono basal como factor patogénico principal en el desarrollo de la ERGE primaria; así, la hipotonía del EEI generada por una pérdida del tono basal o presión de reposo que respondería a factores neurales, hormonales, neurohormonales, de relación paracrina y propios del músculo liso (alrededor de un $25 \%$ de pacientes con ERGE tienen un tono basal similar al de sujetos normales, situación que se podría explicar porque las presiones de reposo del EEI que se miden en condiciones de laboratorio no necesariamente reflejan la capacidad de presión de reposo (tono) habitual del EEI, la que depende de diversos factores como excesos dietéticos, consumo de tabaco, café y alcohol); no obstante ello, se ha visto que pacientes con reflujo clínicamente más grave, esofagitis importante y/o complicaciones de la ERGE suelen tener una hipotonía marcada, con valores iguales o inferiores a $7 \mathrm{mmHg}$. Por su parte, se debe mencionar que la longitud del EEI se encuentra directamente relacionada con la eficiencia mecánica de este, de tal modo que se ha demostrado que la actividad antirreflujo de un EEI normotónico pero corto es nula; es así como la longitud del EEI juega un rol relevante en la resistencia al reflujo causado por incrementos de la presión intra abdominal de diversa naturaleza (obstrucción al flujo distal, aerofagia, etc.). En estudios de monitoreo continuo del EEI se ha observado la existencia de relajaciones espontáneas y transitorias (RET) de corta duración, responsables del $98 \%$ de los episodios de reflujo en sujetos con presiones normales del EEI; no obstante ello, se ha visto que en pacientes con ERGE, las RET pueden durar entre 5 y $30 \mathrm{~s}$, y pueden acompañarse de episodios de reflujo en el $60 \%$ de los casos; además en la mayor parte de las RET no se registra una secuencia peristáltica normal que permita eliminar el líquido refluido al esófago. Otro elemento que hay que considerar es el incremento de la presión intra abdominal, la que puede ocurrir durante el ejercicio, maniobras de valsalva, con el desarrollo del embarazo y el crecimiento de tumores abdominales. Por otra parte, se ha de tener presente el importante rol que juega el aclaramiento esofágico, en el que actúan la fuerza de gravedad, la actividad motora del esófago, la salivación y la fijación del esófago distal en el abdomen; que es el mecanismo encargado de barrer el remanente del 
bolo alimenticio que no alcanza a llegar al estómago luego de la deglución y al barrido de material refluido desde el estómago. Además, existe un interesante concepto referente a los distintos tipos de reflujo; es así como se habla de reflujo primario cuando existe una falla idiopática del EEI y de reflujo secundario cuando existe un incremento de la presión intra abdominal capaz de sobreponerse al tono de reposo del EEI (síndrome pilórico, neoplasias gástricas, intestinales, embarazo, etc.) ó cuando ocurre como complicación evolutiva dentro de la historia natural de una enfermedad sistémica (esclerodermia, diabetes mellitus, distrofia muscular, etc.). En relación a la composición del líquido refluido, hay que recordar que éste se constituye por bolo alimenticio, la secreción gástrica y el contenido duodenal; y cada uno de estos componentes puede favorecer la aparición de reflujo; por ejemplo: la cantidad de lo ingerido se asocia con el incremento de presión intra gástrica; la calidad de lo ingerido puede agravar el reflujo por tratarse de sustancias irritantes para la mucosa esofágica y por activadores de hormonas que relajan el EEI o aumentan la producción de ácido, agravan por ende el reflujo y sus consecuencias; la secreción de ácido y pepsina son el factor más relevante en la producción de la esofagitis por reflujo; algunos componentes de la bilis y la secreción pancreática presentes en el contenido duodenal (sales y ácidos biliares y la tripsina) pueden ser determinantes en el potencial de daño a la mucosa esofágica y patogenia de la metaplasia de Barrett y el eventual desarrollo ulterior de focos de displasia y neoplasia del tipo adenocarcinoma. Finalmente, cabe mencionar que la respuesta de la mucosa esofágica al reflujo es muy variable, de tal forma que existen sujetos que de forma independiente de que sean o no sintomáticos, ante un mismo nivel de exposición de la mucosa esofágica al ácido, desarrollan o no esofagitis y de diversa magnitud y gravedad (ACG; AEG; Armastrong et al.; Engström et al., 2005).

\section{CUADRO CLÍNICO Y DIAGNÓSTICO}

La ERGE presenta un amplio espectro de formas de presentación, lo que la hace aún más compleja. Las manifestaciones clínicas se han ido precisando con el tiempo. Desde un punto de vista esquemático, los síntomas de la ERGE se han dividido en típicos o de origen digestivo; y atípicos o de origen extra digestivo.

\section{Cuadro clínico}

Entre los síntomas típicos o de origen digestivo se encuentran pirosis, regurgitación, disfagia, eructo fácil, sialorrea, hipo o singulto. La pirosis se manifiesta como sensación de ardor que asciende desde el epigastrio hasta el cuello, por detrás del esternón (pirosis retro esternal ascendente), de duración variable (desde minutos hasta horas), de diversa intensidad (desde sensación de ardor hasta dolor intenso), pudiendo extenderse hasta la garganta, en especial cuando se adoptan posturas que facilitan el reflujo (al agacharse, al acostarse en decúbito dorsal, cuando se produce aumento de la presión intra gástrica, en el transcurso del embarazo, etc.). Históricamente, se ha asociado a la pirosis como un síntoma patognomónico de ERGE y constituye la traducción clínica del contacto del material refluido con la mucosa esofágica, se encuentre esta inflamada o no (Manterola et al., 1998).

Por su parte, la regurgitación corresponde a la percepción de la llegada de un líquido ácido o amargo a la boca y es la traducción clínica de la presencia del líquido refluido en la faringe e incluso en la cavidad oral sin que haya sido mediado por vómitos; de hecho, en ciertas ocasiones, se refiere como la sensación de recibir en la hipofárinx material alimentario recién ingerido. La cuantía del material refluido puede ser variable, desde la impresión de acidez en la boca, hasta regurgitaciones de gran cantidad que pueden hacer necesaria su expulsión al exterior, o de lo contrario pueden pasar a las vías respiratorias causando tos, disnea e incluso neumonitis por aspiración cuando esto se produce en condiciones de decúbito supino (ACG).

La disfagia es la sensación de dificultad al paso de los alimentos por el esófago, la que puede ser incluso dolorosa. Este síntoma puede traducir la existencia de complicaciones como esofagitis, estenosis del lumen 
esofágico y aparición de un trastorno motor del cuerpo esofágico secundario a la ERGE. Suele aparecer después de una larga historia de ERGE, se caracteriza por ser de carácter intermitente y a alimentos sólidos (AEG).

El eructo fácil y la flatulencia corresponden a la expulsión de gas proveniente del estómago por la boca; este fenómeno, se debe a un exceso de aire deglutido, con motivo del incremento de la salivación que intenta neutralizar el ácido refluido. Suele ser más frecuente en períodos postprandiales y de forma ocasional, estos eructos pueden ser dolorosos. Aunque el eructo es un mecanismo fisiológico que representa la relajación del EEI para permitir la eliminación de gas desde el fondo gástrico, en pacientes con ERGE puede ser la manifestación de de un EEI hipotónico, de RET frecuentes del EEI, o de una alteración del vaciamiento gástrico (AEG).

La sialorrea se define como salivación permanente. Esta manifestación es frecuente en pacientes con ERGE en relación con los períodos prandial y postprandial, y corresponde a un mecanismo defensivo para optimizar el aclaramiento esofágico en períodos de mayor exposición de la mucosa esofágica al ácido (Armstrong et al.).

El hipo o singulto corresponde a contracción diafragmática espontánea y a veces dolorosa. En pacientes con ERGE, se presenta en relación con los períodos prandial y postprandial; sin embargo, en ocasiones se puede presentar sin relación con la ingesta de alimentos y puede ser de larga duración hasta ceder de forma espontánea (ACG).

Entre los síntomas atípicos o de origen extra digestivo se encuentran los síntomas respiratorios, los síntomas otorrinolaringológicos, el dolor torácico, la anemia, caries dentales, etc. Dentro de los síntomas respiratorios atribuidos a la ERGE se encuentran tos nocturna de carácter crónico, cuadros asmatiformes, neumonitis recurrente y espasmos glóticos. Estos ocurren por el daño a las vías respiratorias causado por el material refluido fundamentalmente durante el sueño, período en el cual el paciente no tiene conciencia ni percibe manifestaciones clínicas, hecho que lo hace atribuir sus síntomas a cuadros respiratorios altos o fenómenos alérgicos.
Ocurren debido al contacto de las mucosas expuestas con el material refluido, o por un reflejo de broncoconstricción. En ocasiones, la gravedad de los cuadros respiratorios puede determinar el desarrollo de daño pulmonar irreversible (Fass et al., 2004; Harding, 2005).

Entre los síntomas otorrinolaringológicos se describen la ronquera matinal, laringitis a repetición, fenómenos de faringitis y otitis media reiterativa. Su génesis está asociada también a la irritación crónica que causa a estos niveles el material refluido desde el estómago (Gatta et al., 2007).

Se he reportado que cerca de un $20 \%$ de los pacientes con ERGE, la enfermedad se manifiesta como un cuadro de dolor precordial de tipo anginoso, con una duración superior a $20 \mathrm{~min}$ y sin irradiación. El dolor suele tener una relación temporal con los síntomas habituales de ERGE o con la deglución; aparece por lo general en decúbito y mejora al ponerse de pie, con la ingestión de antiácidos o al eructar. Es habitual que se presente en reposo, pero que el esfuerzo también puede desencadenar reflujo y causar un dolor idéntico al ángor. Cuando existe una evidente relación con síntomas típicos de la ERGE, el dolor torácico puede ser fácilmente reconocido como de origen esofágico. Sin embargo, existe un grupo de pacientes en los que se presenta como enfermedad cardiaca, y en quienes solo se llega al diagnóstico de ERGE después de haber sido descartado el origen cardiaco del dolor. El hecho de que el dolor calme con la administración de nitritos, no ayuda a distinguir entre dolor cardíaco y esofágico, ya que estos fármacos son capaces de relajar tanto la musculatura lisa del esófago como la de las arterias coronarias. Por esta razón, se hace necesario recurrir además del estudio cardiológico, a diversos estudios esofágicos para lograr así una mayor precisión diagnóstica. Una complicación adicional de este síntoma, es que siendo originado en el esófago, puede estar en relación con alteraciones motoras secundarias a la ERGE, o a trastornos motores esofágicos primarios, como peristalsis esofágica sintomática, espasmo difuso del esófago, EEI hipertensivo y trastornos motores inespecíficos (Manterola et al., 2004).

En pacientes con ERGE se generar anemia hipocroma y normocrómica, por pérdidas 
constantes pero microscópicas de sangre secundarias a erosiones o úlceras de la mucosa esofágica. Esta anemia suele pasar desapercibida en la mayor parte de los pacientes con esofagitis, pero en ocasiones se puede manifestar en forma clínica y evidente a través de una hemorragia digestiva alta, especialmente cuando existen úlceras esofágicas o erosiones extensas (AEG).

\section{Diagnóstico}

No obstante la variada gama de manifestaciones clínicas, cuando se plantea la hipótesis diagnóstica de ERGE con o sin desarrollo de complicaciones evolutivas, es necesario realizar algunas pruebas diagnósticas orientadas a confirmar la hipótesis diagnóstica, evaluar la magnitud y gravedad del fenómeno y la eventual existencia de complicaciones; de forma tal de adoptar una decisión terapéutica basada en evidencias objetivas.

Existe una serie de alternativas de estudio para estos pacientes: escalas de valoración clínica, radiología contrastada, uso radioisótopos, endoscopía, biopsias, manometría esofágica, registro ambulatorio de $\mathrm{pH}$ intra esofágico de $24 \mathrm{~h}$, determinación óptica de reflujo biliar (AEG).

Existen varias escalas de valoración clínica para diagnóstico de ERGE; sin embargo, la mayor parte de ellas carece de estudios de validez y confiabilidad. Nosotros desarrollamos una escala que fue sometida a estudios de validez y confiabilidad que tiene una capacidad discriminatoria superior al $93 \%$; está compuesta por 7 ítems que corresponden a síntomas de reflujo a los que se mide su existencia y frecuencia de aparición, generándose finalmente una sumatoria que puede variar entre 0 y 13 puntos, considerándose como punto de corte para diagnóstico de ERGE, los 3 puntos (Manterola et al., 2002).

La radiología contrastada esófago, gastroduodenal permite por una parte obtener información adicional sobre el tubo digestivo alto, poner de manifiesto la existencia de reflujo espontáneo (para lo cual tiene una baja sensibilidad); detectar complicaciones como estenosis, pesquisar anomalías motoras o sospechar

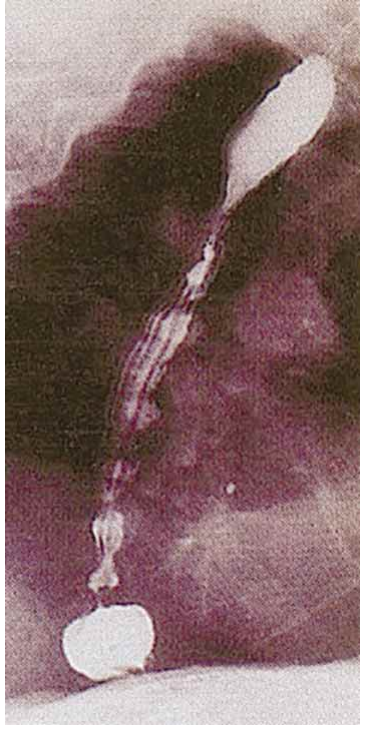

Fig. 2. Radiología contrastada esófago gástrica en la que se verifica una hernia hiatal por deslizamiento.

su existencia; pero sin lugar a dudas, es la mejor prueba para determinar la existencia de una hernia de hiato (ACG) (Fig. 2).

La endoscopía es un excelente método diagnóstico para valorar el estado de la mucosa esofágica y la eventual inflamación secundaria a la ERGE, contando además con la ventaja que nos permite obtener información del resto del tubo digestivo alto y de acceder a muestras de la mucosa esofágica. La sensibilidad de la endoscopía para el diagnóstico de ERGE es baja; sin embargo, cuando nos permite detectar lesiones inequívocas de esofagitis tales como friabilidad espontánea con hemorragia, erosiones, ulceraciones lineales, úlceras profundas y

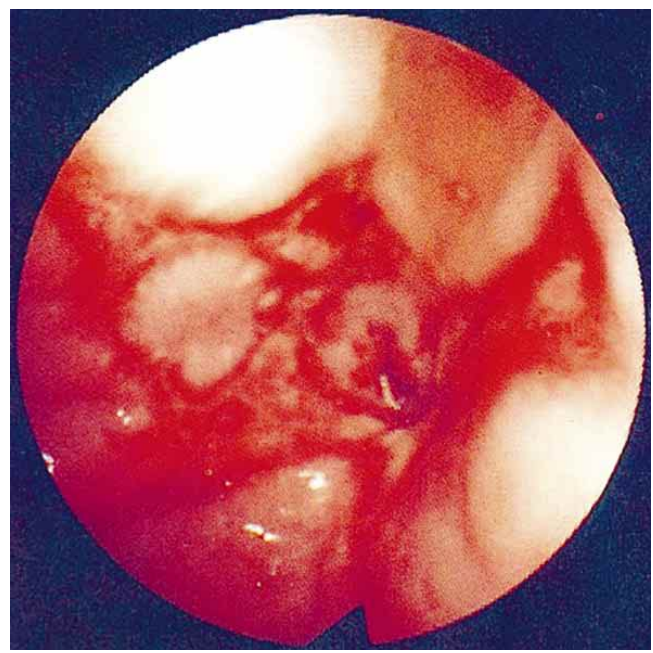

Fig. 3. Endoscopia digestiva alta que permite evidenciar el esófago distal friable y con erosiones. 
estenosis, la sensibilidad y especificidad de esta prueba diagnóstica es muy elevada. Por otra parte, este método permite la realización de maniobras terapéuticas como dilataciones para el tratamiento de la estenosis secundaria a reflujo (AEG) (Fig. 3).

Por su parte, la toma de muestras de mucosa esofágica para su ulterior examen histológico es muy relevante en este tipo de pacientes debido entre otras cosas a que alrededor de un $60 \%$ de las endoscopías en pacientes sintomáticos de reflujo son normales, apareciendo en ellos esofagitis crónica inespecífica en el análisis histológico de las muestra de mucosa esofágica. Por otro lado, es la única forma de plantear el diagnóstico de metaplasia de Barrett, displasia de diversa gravedad y neoplasia. Desde un punto de vista de eficiencia del método, se recomienda obtener entre 2 y 6 biopsias, las que han de obtenerse aproximadamente entre 4 y $5 \mathrm{~cm}$ por encima de la línea $Z$, ya que a veces en la porción más distal del esófago en sujetos normales también pueden aparecer cambios histológicos mínimos de esofagitis seguramente secundarios a los episodios de reflujo postprandiales. La mayor utilidad de la biopsia esofágica es en sujetos con síntomas de ERGE sin esofagitis, con inflamación mínima, - cuando se sospecha una metaplasia de Barrett. Por ende, la mayor ventaja de la endoscopía es incrementar la sensibilidad y especificidad de la endoscopía comoestudio

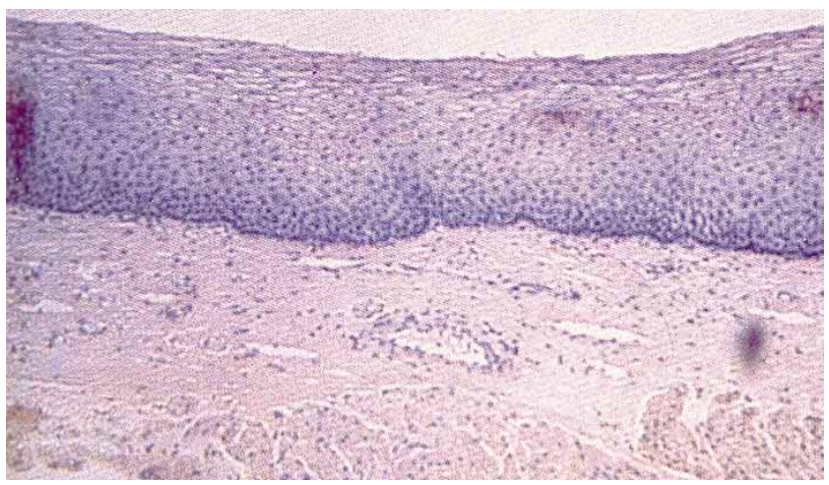

Fig. 4. Imagen histológica de una muestra de un sujeto con esofagitis. Se aprecia la mucosa, constituida por epitelio pavimentoso estratificado que descansa sobre una membrana basal con hiperplasia celular (pleomorfismo nuclear e hipercromatismo) y lámina propia rica en células linfoides. adicional (Fig. 4). Su desventaja radica en una menor especificidad que la endoscopia, dada por el hecho que en el lugar de donde se obtienen las muestras puede no existir inflamación, debido a la orientación espacial y el ángulo en el que se toman las muestras (Armstrong et al.).

La manometría esofágica no es un método diagnóstico de ERGE. Esta, aporta datos más bien indirectos, como el estudio del EEI (presión de reposo, longitud, morfología y respuesta deglutoria), del cuerpo esofágico (presión de reposos, amplitud y duración de las ondas peristálticas y presencia de ondas secundarias y terciarias) y del EES (presión de reposo, Iongitud, respuesta deglutoria y sinergia faringoesofágica). En relación al EEI, como ya se mencionó, la verificación de una hipotonía esfinteriana marcada y un EEI corto, son hechos relevantes pues en conjunto con la detección de hipoperistalsis o trastorno motor del cuerpo esofágico, y alteraciones presivas relevantes del EES, son orientadores de la magni-

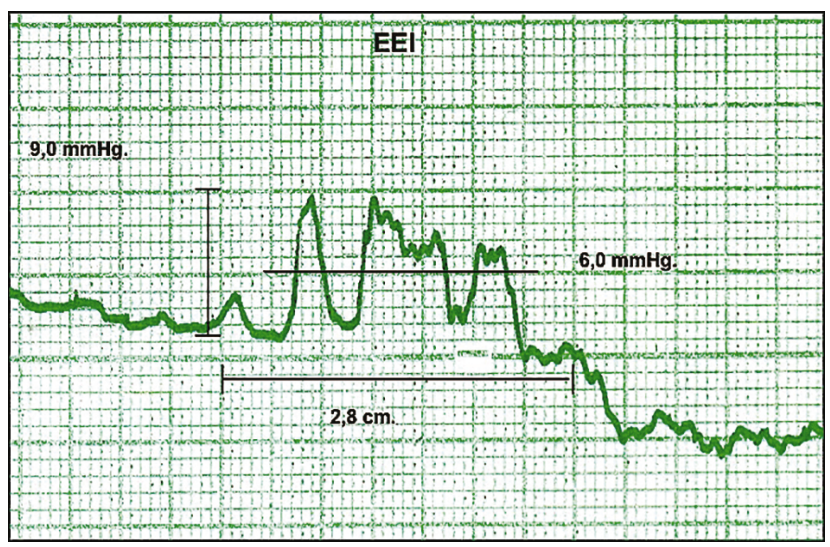

Fig. 5. Registro manométrico de un EEI hipotónico, de $6 \mathrm{mmHg}$ de tono basal y $2,8 \mathrm{~cm}$ de longitud.

tud del problema y por ende nos orientan acerca del pronóstico de la enfermedad y de sus potenciales alternativas terapéuticas. Por último, la manometría es importante para determinar la localización del EEI y de esta forma instalar el electrodo de registro de la pHmetría esofágica de 24 h (Manterola et al., 1998, 2000, 2001a, 2001b) (Fig. 5).

El registro ambulatorio del $\mathrm{pH}$ intra esofágico consiste en la medición durante $24 \mathrm{~h}$ del $\mathrm{pH}$ del tercio distal del esófago, de forma tal 
que cada vez que se produzca paso de contenido gástrico al esófago, variará el $\mathrm{pH}, \mathrm{y}$, este cambio será susceptible de ser registrado. Esta exploración cuantifica de forma fisiológica la presencia de reflujo, permite correlacionar la sintomatología con los cambios del $\mathrm{pH}$ y además es un reflejo de la capacidad de aclaramiento esofágico. Los electrodos, unidos a un aparato con memoria electrónica portátil permiten el registro de los cambios del $\mathrm{pH}$, los que posteriormente son analizados en un computador mediante programas informáticos, desde los cuales se emite un informe final. La pHmetría ambulatoria de $24 \mathrm{~h}$, se considera en la actualidad la prueba de mayor eficiencia diagnóstica para ERGE, por su elevada sensibilidad y especificidad. Durante el desarrollo de la prueba, se pretende reproducir al máximo las condiciones normales de vida de los pacientes, por lo que no se restringe ningún tipo de comida, bebida o hábito de vida, aún cuando no se administra medicación alguna que pueda modificar o alterar el resultado del registro (antiácidos, inhibidores $\mathrm{H} 2$ o de la bomba de protones). La mayor utilidad de la pHmetría ambulatoria en población adulta, se obtiene en aquellos pacientes con síntomas atípicos de ERGE (dolor torácico, síntomas respiratorios, etc.); con síntomas de ERGE sin respuesta al tratamiento médico o en los que no se ha podido objetivar la existencia de reflujo mediante otros medios; pacientes con ERGE e indicación quirúrgica; con recidiva de los síntomas de ERGE tras cirugía antirreflujo; con síntomas crónicos de ERGE, asociado o no a síntomas respiratorios. Por otro lado, se ha de considerar la utilidad de la pHmetría en el control y evaluación de la tera-

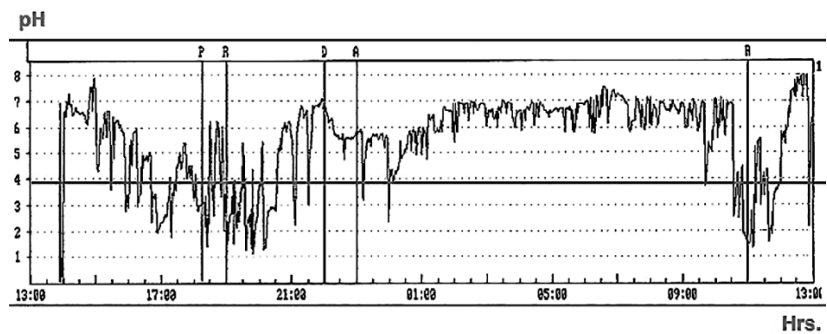

Fig. 6. Registro ambulatorio de pH intra esofágico de 24 horas, en el que se verifican múltiples episodios de reflujo ácido (bajo la línea de $\mathrm{pH} 4$ ). La mayor parte de ellos en períodos post prandiales, y asociados con el registro de los síntomas pirosis $(P)$ y regurgitación $(R)$. No se constató asociación entre episodios de reflujo y los síntomas disfagia (D) y ahogo (A). péutica empleada, especialmente en aquellos pacientes que son sometidos a cirugía antirreflujo, en los que esta prueba constituye el "control de calidad" del procedimiento efectuado (Fig. 6) (Manterola et al., 2001a, 2001b).

El monitoreo óptico de reflujo biliar consiste en un sistema de registro ambulatorio para detectar la presencia de bilirrubina en el interior del esófago, como marcador de reflujo duodenal. Esto, se realiza mediante un método espectrofotométrico. La bilirrubina tiene una absorbancia específica, que es captada a través de electrodo especial, almacenada en una unidad de registro similar a un pHmetro y posteriormente elaborada la información en un ordenador, mediante programas informáticos. Es un método que aún cuando está en evaluación, ha permitido conocer algo más acerca de la fisiopatología de la ERGE y la asociación de esta con el desarrollo de metaplasia de Barrett. Tanto es así, que en análisis de amplios grupos de pacientes con ERGE se ha podido establecer que en cerca del $60 \%$ de los individuos con ERGE la presencia de bilirrubina sobrepasa el dintel establecido como normal, hecho que sugiere que la presencia de bilirrubina en el esófago parece tener relación directa con el daño mucoso y ésta presencia se hace especialmente relevante en los pacientes con esófago de Barrett. Las desventajas de este método son que bilirrubina es sólo un componente del líquido refluido y como tal se utiliza como marcador, pero no presupone su efecto nocivo, el que queda patente en sustancias como ácidos biliares, enzimas pancreáticos y la lisolecitina que este instrumento no mide, pero además: Existe una gran variabilidad de la concentración de bilirrubina en la bilis; la variabilidad también es evidente para la concentración de bilis en el líquido refluido (ACG).

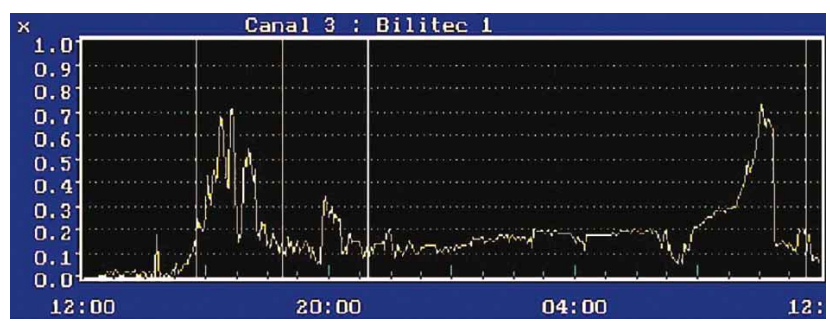

Fig. 7. Registro ambulatorio de monitoreo óptico de reflujo biliar de 24 horas, en el que se detecta la presencia de bilirrubina en dos episodios post prandiales claramente visibles (entre las 16:00 h y 18:00 h; y entre las 09:00 h y 11:00 h). 
La falta de homogeneidad del contenido gástrico, que incluye moco y partículas sólidas puede interferir la lectura. Por último, el equipamiento utilizado, confeccionado con fibra óptica posee indiscutibles limitaciones y duración (Fig. 7).

\section{TRATAMIENTO Y PRONÓSTICO}

Plantear estrategias de tratamiento para pacientes con ERGE es un desafío, pues a pesar de que los distintos grupos de trabajo han desarrollado algoritmos y consensos respecto de este controversial punto, sabemos que en esta materia no se puede generalizar, debido a que cada paciente responde de forma diferente, razón por la que se estima que se ha de aplicar estrategias diferentes para cada paciente; debido a la multiplicidad de variables anatómicas, fisiológicas y fisiopatológicas, particulares de cada individuo que lo hacen diferente de otro (ACG; AEG).

No obstante ello, en términos generales, se puede decir que el tratamiento persigue esencialmente proporcionar alivio sintomático y curación de eventuales lesiones endoscópicas e histológicas; por lo que de forma esquemática se puede resumir en medidas generales y dietéticas, tratamiento médico o farmacológico y tratamiento quirúrgico (Figs. 8,9 y 10).

Las medidas higiénicas, posturales y dietéticas componen el pilar fundamental del tratamiento de la ERGE, en términos de un cambio en el estilo de vida del paciente, el que debe comprender el carácter crónico y recurrente de los síntomas, los que pueden reaparecer en relación con excesos alimenticios y esfuerzos físicos. Las medidas higiénicas y posturales incluyen disuadir a los pacientes de subir de peso, de dormir recién comido, de hacer ejercicios bruscos o deportes recién comido y de utilizar ropas apretadas; comer cantidades reducidas de alimento y de forma frecuente, de tal forma de dividir la ración diaria en 6 o 7 comidas en vez de 2 o 3; hacer la última comida de la noche como mínimo 2 a $3 \mathrm{~h}$ antes de acostarse a dormir y dormir con la cabecera de la cama elevada respecto al resto de la cama. Las medidas dietéticas incluyen la restricción en el consumo de bebidas alcohólicas (incluyendo la cerveza), bebidas gaseosas (especialmente las del tipo cola), chocolate y sus derivados, café, tabaco,

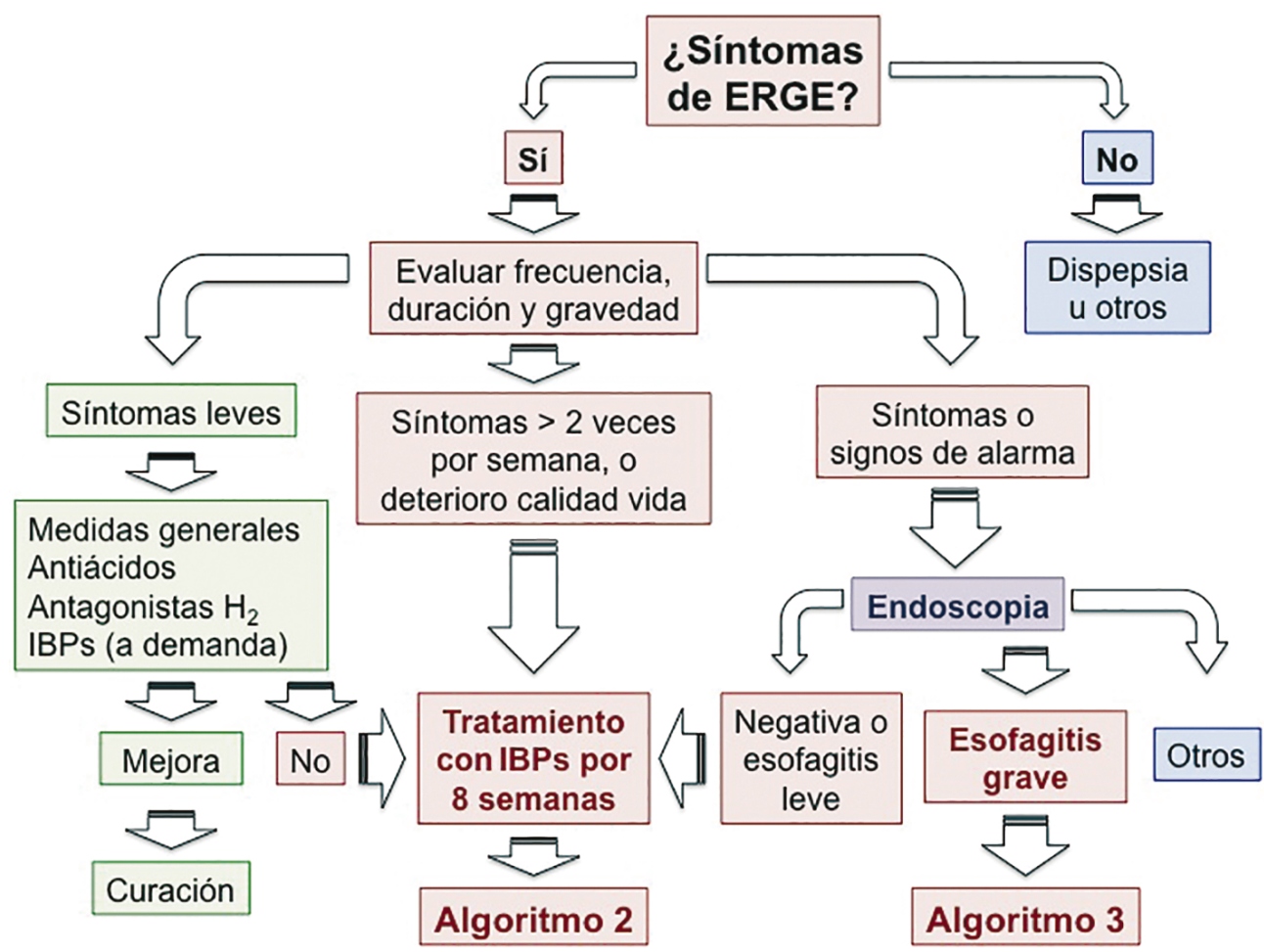

Fig. 8. Algoritmos diagnóstico terapéuticos. Qué hacer en presencia de síntomas de ERGE. 


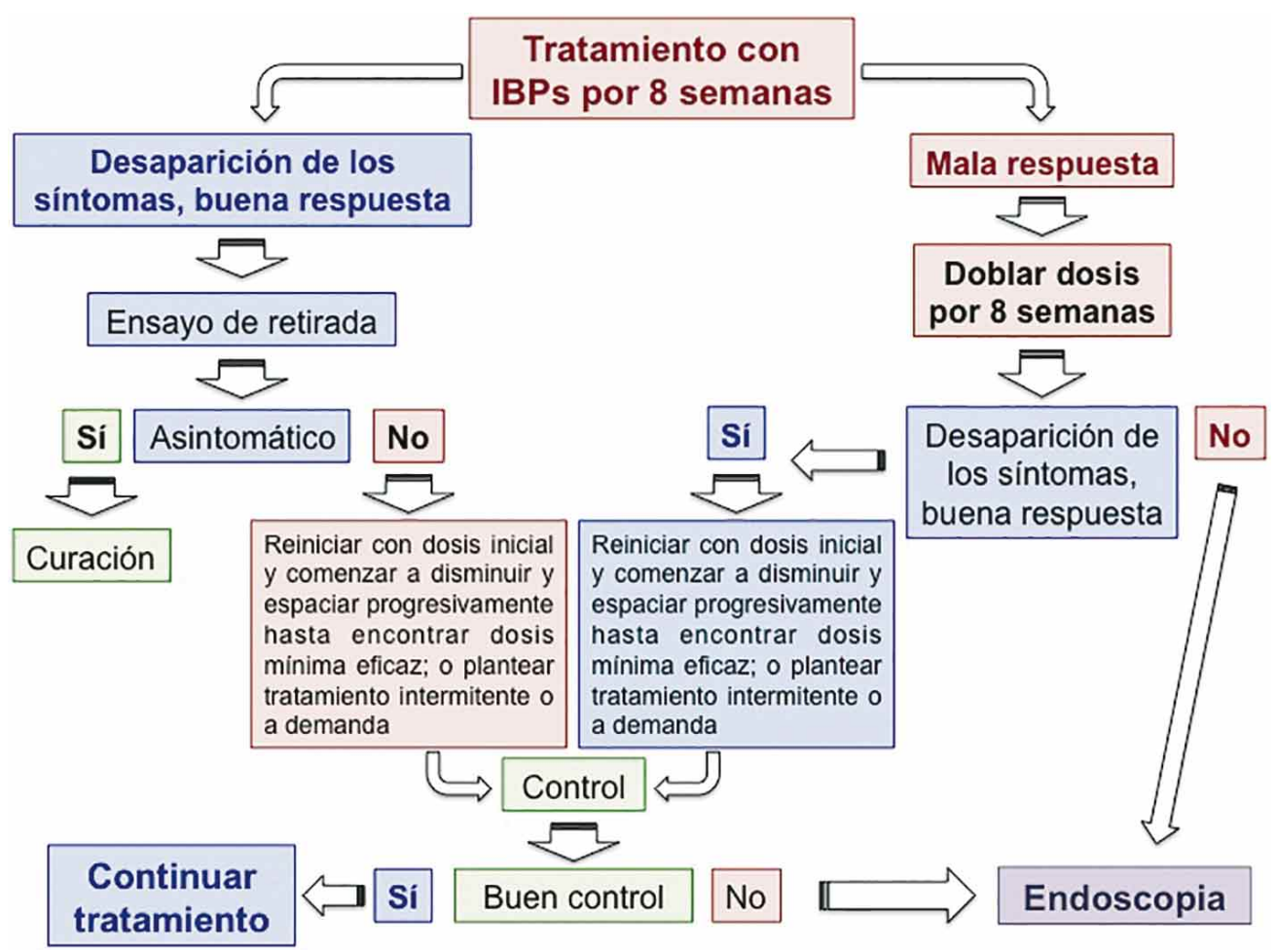

Fig. 9. Algoritmos diagnóstico terapéuticos. Cómo continuar una vez instaurado el tratamiento con IBPs.

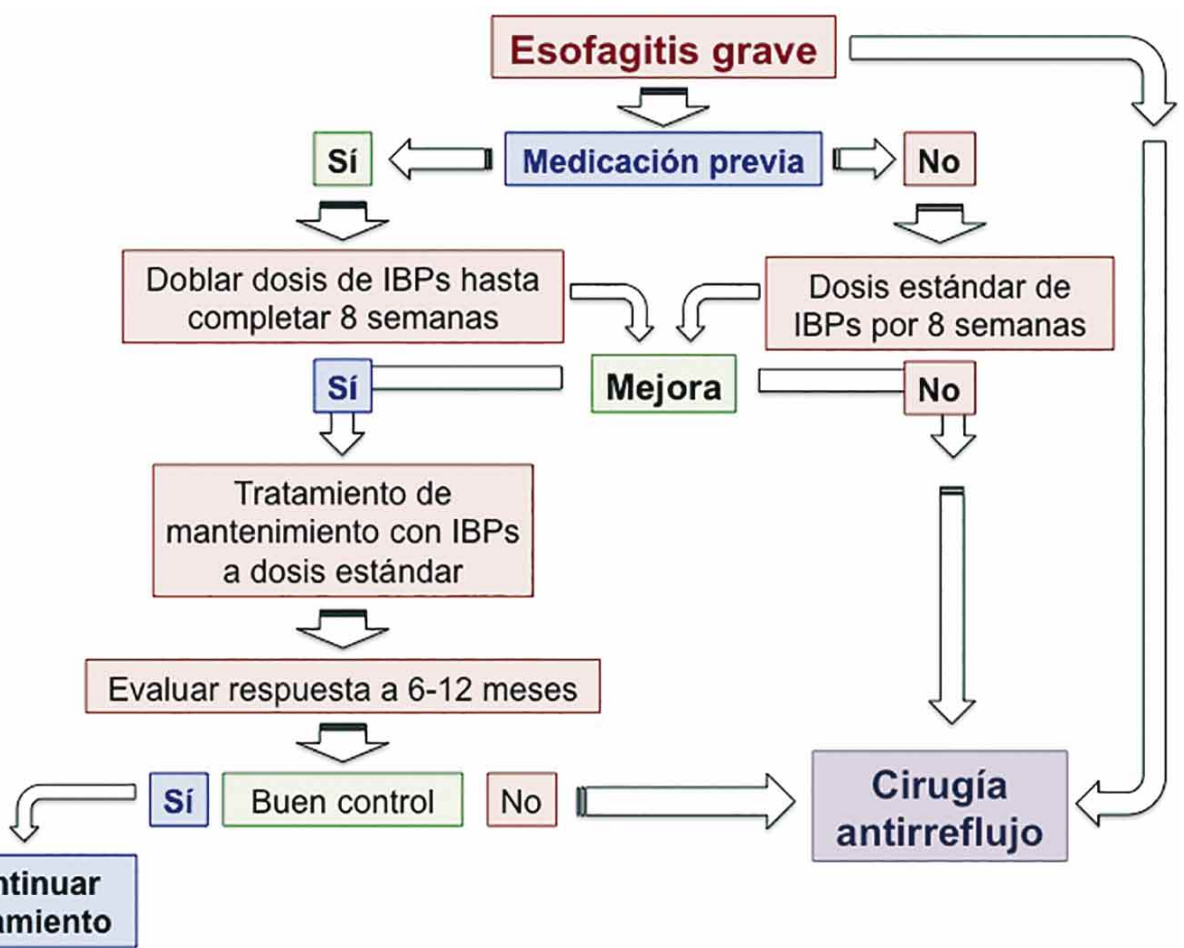

Fig. 10. Algoritmos diagnóstico terapéuticos. Tratamiento de la esofagitis grave. 
carminativos como la menta, frituras y alimentos ricos en grasas (algunas de estas sustancias generarían un incremento de la secreción ácido-péptica del estómago; otras, una acción irritante sobre la mucosa esofágica, secundaria a hiperosmolaridad; y otras, un efecto hipotensor sobre el EEI (AEG).

Además, se ha de mencionar el efecto de algunos fármacos con efecto irritante directo sobre la mucosa esofágica y gástrica; y otras con efecto hipotensor sobre el EEI como antiinflamatorios no esteroidales, cortico esteroides, productos hormonales femeninos, bloqueadores de los canales de calcio, agonistas Badrenérgicos, sedantes e hipnóticos, teofilinas, etc., razón por la que están contraindicados en pacientes con ERGE.

El tratamiento médico o farmacológico incluye antiácidos, alginatos, procinéticos (domperidona y metoclopramida), bloqueadores de los receptores $\mathrm{H} 2$ de histamina (famotidina), e inhibidores de la bomba de protones o IBPs (omeprazol, lanzoprazol, rabeprazol y esomeprazol). Los antiácidos han sido desde siempre los fármacos más utilizados para el control de los síntomas de la ERGE, debido al rápido alivio de síntomas que producen; el que se asocia a la neutralización del componente ácido del jugo gástrico y eventualmente a un efecto de absorción de ácidos biliares. Una variedad de estos es la asociación de antiácidos con alginatos, que actuarían generando una capa flotante de espuma viscosa que impediría el reflujo. Los procinéticos son un grupo de fármacos cuya eficiencia está muy cuestionada, pues aunque la mayor parte de estos aceleran el vaciamiento gástrico, no hay evidencia acerca de su efecto sobre la peristalsis esofágica, menos aún sobre el tono del EEI. Los bloqueadores de los receptores $\mathrm{H} 2$ de histamina, son capaces de reducir entre un $60 \%$ y un 70 $\%$ la secreción ácida basal en dosis habituales; por ende permiten un apropiado control de los síntomas, sin embargo esto no suele asociarse a una mejoría endoscópica de las lesiones; son efectivos como tratamiento de esofagitis pero requiere de mayor tiempo y la tasa de recidiva es mayor. Por su parte, los IBPs en dosis convencionales, son capaces de reducir en más de un $90 \%$ de la secreción ácido-péptica del estómago por lo que son obviamente más eficien- tes que los bloqueadores de los receptores $\mathrm{H} 2$ en el control de síntomas de la ERGE; a pesar de ello, las tasa de recidiva sintomática y de esofagitis endoscópica son significativas una vez suspendido su uso; hecho que, asociado al elevado coste de éstos (especialmente en sujetos que requieren de uso en forma crónica), hacen plantear la necesidad de una intervención quirúrgica como solución definitiva para la ERGE, a menos que exista una contraindicación formal (Dore et al., 2007; Galindo et al., 2013; Glatzel et al., 2007; Hawkey et al., 2007; Kahrilas et al., 2005; Kim et al., 2006; Miner et al., 2003; Scholten et al., 2007; Sigterman et al., 2013; Vcev et al., 2006).

Finalmente, la cirugía juega un rol relevante en cualquier algoritmo terapéutico de ERGE. Las técnicas quirúrgicas que se han utilizado a lo largo de la historia para el tratamiento de la ERGE y como de sus complicaciones son diversas; sin embargo, actualmente la más utilizada es la fundoplicatura descrita por Nissen, la que a su vez se puede realizar por vía abierta y laparoscópica en sus distintas variantes, las que pretenden reestablecer un segmento de alta presión en la unión esófagogástrica para reforzar la acción del EEI y restablecer el segmento intra abdominal del esófago; existiendo reportes de efectividad de hasta un $92 \%$ en términos de desaparición de síntomas de reflujo a 20 años de seguimiento. Las indicaciones quirúrgicas actuales en ERGE basadas en una extensa revisión de la evidencia disponible; y, una vez descartadas las contraindicaciones formales de cirugía; se podrían resumir en cuatro situaciones: en Fallo de tratamiento médico, entendido como recurrencia sintomática frecuente, persistencia de síntomas durante un tratamiento controlado o intolerancia a la terapia, y la persistencia de regurgitación de alto volumen a pesar del tratamiento con farmacológico; pacientes con esofagitis erosiva a repetición, o que se mantiene a pesar del tratamiento conservador, situación para la que existe evidencia suficiente que permite avalar de forma objetiva que la recidiva de una esofagitis tras un tratamiento farmacológico de varias semanas (incluso con IBPs), es del $50 \%$ al $80 \%$ a 1 año, razón por la cual se precisa mantener el tratamiento para prevenir su reaparición; pacientes con síntomas atípicos de ERGE que remiten con trata- 
miento antisecretor, pues existe evidencia suficiente acerca de la asociación de la ERGE y algunos problemas respiratorios (asma, neumonías por aspiración y tos crónica), otorrinolaringológicos (laringitis crónica) y dolor torácico recurrente; existencia de complicaciones (úlcera esofágica, estenosis y esófago de Barrett); y cuando existe un deseo expreso del paciente, situación que se sustenta en que el coste del tratamiento anti secretor a largo plazo, el temor a desarrollar potenciales efectos colaterales secundarios al consumo crónico de medicamentos anti secretores como los IBPs y el escaso nivel de adherencia a tratamientos prolongados, tesitura, en la que debe tenerse en cuenta el estado psicológico del paciente, pues existen evidencias que orientan a que un porcentaje significativo de sujetos con ERGE presentan trastornos psiquiátricos que afectan en forma notable el resultado de la cirugía antirreflujo (Chrysos et al., 2004; Pessaux et al., 2005; Draaisma et al., 2006; Håkanson et al., 2007; Lundell et al.; Garg \& Gurusamy, 2015).

Dentro de las opciones quirúrgicas, existen ensayos clínicos aleatorios y estudios de cohortes (evidencia 1 b y $2 b$ ), que permiten sostener que la fundoplicatura, sea total o parcial, se realice por vía abierta o laparoscópica, ofrece un apropiado control de síntomas y la prevención de esofagitis a mediano y largo plazo, con una baja tasa de morbilidad y mortalidad que en equipos con experiencia no superan el 5 $\%$ y $0,5 \%$ respectivamente. Además, ha demostrado su mayor coste-beneficio y coste-utilidad. Sin embargo debe ponerse especial énfasis a que los buenos resultados sólo se logran si se definen con claridad las indicaciones y se informa a los pacientes de forma detallada de los resultados previsibles, que incluye de forma necesaria los posibles efectos secundarios de la intervención (Salminen et al., 2007; Lundell et al.; Håkanson et al.).

Por lo antes expuesto, una propuesta de algoritmo de tratamiento es que en una primera etapa, indicar la modificación de los hábitos de vida del paciente, asociando la administración de antiácidos y/o procinéticos; en una segunda etapa, introducir inhibidores de la secreción gástrica (bloqueadores de los receptores $\mathrm{H} 2$ de histamina e IBPs); y en una tercera etapa, si las medidas anteriores han demostrado ser ineficientes en el control de los síntomas, o es necesario mantener dosis elevadas de IBPs durante lapsos de tiempos prolongados, indicar cirugía antireflujo.

MANTEROLA, C. Gastroesephageal reflux disease. Int. J. Med. Surg. Sci., 3(1):795-809, 2015.

SUMMARY: Gastroesophageal reflux disease (GERD) is one of the most frequent causes of consultation in primary care and gastroenterology. Over $40 \%$ of the general population experiences occasional heartburn and regurgitation due to GERD. It is a complex disease and physiology and pathogenesis are not yet completely defined. However, abnormalities in the lower esophageal sphincter, esophageal motility or gastric emptying can cause or worsen GERD. There are various diagnostic and therapeutic approaches; however, the use of proton-pump inhibitor fundoplication permanence and (antireflux surgery), are the most effective options.

KEY WORDS: Gastroesophageal reflux disease; Gastroenterology; Esophageal complications; Endoscopically.

\section{REFERENCIAS BIBLIOGRÁFICAS}

American College of Gastroenterology (ACG). Web Site. 2016. Disponible en: http://www.acg.gi.org/ acg-dev/staging/members/guides/gerdabs.html.

Armstrong, D.; Marshall, J. K.; Chiba, N. ; Enns, R.; Fallone, C. A. ; Fass, R.; Hollingworth, R.; Hunt, R. H.; Kahrilas, P. J.; Mayrand, S.; Moayyedi, P.; Paterson, W. G.; Sadowski, D.; van Zanten, S. J. \& Canadian Association of GastroenterologyGERD Consensus Group. Canadian Consensus Conference on the management of gastroesophageal reflux disease in adults - update 2004. Can. J. Gastroenterol., 19(1):15-35, 2005.

Asociación Española de Gastroenterología (AEG). Guía de Práctica Clínica. Web Site. 2016. Disponible en: http://www.aegastro.es/docentia/ atencion-primaria/guias-practica-clinica 
Avidan, B.; Sonnenberg, A. ; Giblovich, H. \& Sontag, S. J. Reflux symptoms are associated with psychiatric disease. Aliment. Pharmacol. Ther., 15(12): 1907-12, 2001.

Cameron, A. J.; Lagergren, J.; Henriksson, C.; Nyren, O.; Locke, G. R. 3rd. \& Pedersen, N. L. Gastroesophageal reflux disease in monozygotic and dizygotic twins. Gastroenterology, 122(1):55-9, 2002.

Cohen, H.; Moraes-Filho, J. P.; Cafferata, M. L. ; Tomasso, G.; Salis, G.; González, O.; Valenzuela, J.; Sharma, P.; Malfertheiner, P.; Armstrong, D.; Lundell, L.; Corti, R.; Sakai, P.; Ceconello, I. \& Latin-American GORD Consensus Group. Latin-American GORD Consensus Group. An evidence-based, LatinAmerican consensus on gastro-oesophageal reflux disease. Eur. J. Gastroenterol. Hepatol., 18(4):349-68, 2006.

Collings, K. L.; Pierce Pratt, F.; Rodriguez-Stanley, S.; Bemben, M. \& Miner, P. B. Esophageal reflux in conditioned runners, cyclists, and weightlifters. Med. Sci. Sports Exerc., 35(5):7305, 2003.

Chrysos, E.; Athanasakis, E.; Pechlivanides, G.; Tzortzinis, A.; Mantides, A. \& Xynos, E. The effect of total and anterior partial fundoplication on antireflux mechanisms of the gastroesophageal junction. Am. J. Surg., 188(1):39-44, 2004.

Dore, M. P.; Pedroni, A.; Pes, G. M.; Maragkoudakis, E.; Tadeu, V.; Pirina, P.; Realdi, G. ; Delitala, G. \& Malaty, H. M. Effect of antisecretory therapy on atypical symptoms in gastroesophageal reflux disease. Dig. Dis. Sci,,52(2):463-8, 2007.

Draaisma, W. A. ; Rijnhart-de Jong, H. G. ; Broeders, I. A.; Smout, A. J.; Furnee, E. J. \& Gooszen, H. G. Five-year subjective and objective results of laparoscopic and conventional Nissen fundoplication: a randomized trial. Ann. Surg., 244(1):34-41, 2006.

Engström, C.; Ruth, M.; Lönroth, H. \& Lundell, L. Manometric characteristics of the gastroesophageal junction after anterior versus posterior partial fundoplication. Dis. Esophagus, 18(1):31-6, 2005.

Galindo, G.; Vassalle, J.; Marcus, S. N. \& Triadafilopoulos, G. Multimodality evaluation of patients with gastroesophageal reflux disease symptoms who have failed empiric proton pump inhibitor therapy. Dis. Esophagus, 26(5):443-50, 2013.
Garg, S. K. \& Gurusamy, K. S. Laparoscopic fundoplication surgery versus medical management for gastro-oesophageal reflux disease (GORD) in adults. Cochrane Database Syst. Rev., 11:CD003243, 2015

Fass, R.; Achem, S. R. ; Harding, S.; Mittal, R. K. \& Quigley, E. Review article: supra-oesophageal manifestations of gastro-oesophageal reflux disease and the role of night-time gastrooesophageal reflux. Aliment. Pharmacol. Ther., 20 Suppl. 9:26-38, 2004.

Gatta, L.; Vaira, D.; Sorrenti, G.; Zucchini, S.; Sama, C. \& Vakil, N. Meta-analysis: the efficacy of proton pump inhibitors for laryngeal symptoms attributed to gastro-oesophageal reflux disease. Aliment. Pharmacol. Ther., 25(4): 385-92, 2007.

Glatzel, D.; Abdel-Qader, M.; Gatz, G. \& Pfaffenberger, B. Pantoprazole $40 \mathrm{mg}$ is as effective as esomeprazole $40 \mathrm{mg}$ to relieve symptoms of gastroesophageal reflux disease after 4 weeks of treatment and superior regarding the prevention of symptomatic relapse. Digestion, 75 Suppl. 1:69-78, 2007.

Håkanson, B. S.; Thor, K.B.; Thorell, A. \& Ljungqvist, O. Open vs laparoscopic partial posterior fundoplication. A prospective randomized trial. Surg. Endosc., 21(2):289-98, 2007.

Harding, S. M. Gastroesophageal reflux: a potential asthma trigger. Immunol. Allergy Clin. North Am., 25(1):131-48, 2005.

Hawkey, C. J.; Jones, R. H.; Yeomans, N. D.; Scheiman, J. M.; Talley, N. J.; Goldstein, J. L.; Ahlbom, H. \& Naesdal, J. Efficacy of esomeprazole for resolution of symptoms of heartburn and acid regurgitation in continuous users of non-steroidal anti-inflammatory drugs. Aliment. Pharmacol. Ther., 25(7):813-21, 2007.

Kahrilas, P. J.; Miner, P.; Johanson, J.; Mao, L.; Jokubaitis, L. \& Sloan, S. Efficacy of rabeprazole in the treatment of symptomatic gastroesophageal reflux disease. Dig. Dis. Sci., 50(11):2009-18, 2005.

Kim, J.; Ogai, A.; Nakatani, S.; Hashimura, K.; Kanzaki, H.; Komamura, K.; Asakura, M.; Asanuma, H.; Kitamura, S.; Tomoike, H. \& Kitakaze, M. Impact of blockade of histamine $\mathrm{H} 2$ receptors on chronic heart failure revealed by retrospective and prospective randomized studies. J. Am. Coll. Cardiol., 48(7):1378-84, 2006. 
Lundell, L.; Bell, M. \& Ruth, M. Systematic review: Laparoscopic fundoplication for gastroesophageal reflux disease in partial responders to proton pump inhibitors. World $\mathrm{J}$. Gastroenterol., 20(3):804-13, 2014.

Manterola, C.; Calderón, C.; Pérez, O.; Flores, P. \& Hofmann, E. Esofagitis por reflujo gastroesofágico y alteración de párametros presivos esofágico. Acta Méd. Colomb., 23(1): 7-14, 1998.

Manterola, C.; Muñoz, S.; Flores, P.; Fernández, E. \& Capurro, M. Study of association between the magnitude of sphincter hypotonia and esophageal motor disorders. Rev. Med. Chil., 128(7):721-8, 2000.

Manterola Delgado, C.; Gutiérrez Luengo, R.; Soto F., A.; Cuadra M., A.; Maurer D., J.; Moreno L., F. \& Leiva M., M. Modificación de variables clínicas y del laboratorio en pacientes sometidos a cirugía antirreflujo. Rev. Chil. Cir., 53(3):287-92, $2001 a$.

Manterola, C.; Muñoz, S.; Fernández, E. \& Flores, P. Estudio de asociación entre esofagitis endoscópica y deterioro de parámetros motores esofágicos en pacientes con enfermedad por reflujo gastroesofágico. Rev. Chil. Cir., 53(4):362-9, 2001 b.

Manterola, C.; Muñoz, S.; Grande, L. \& Bustos, L. Initial validation of a questionnaire for detecting gastroesophageal reflux disease in epidemiological settings. J. Clin. Epidemiol., 55(10): 1041-5, 2002.

Manterola, C.; Barroso, M. S.; Losada, H.; Muñoz, S. \& Vial, M. Prevalence of esophageal disorders in patients with recurrent chest pain. Dis. Esophagus, 17(4):285-91, 2004.

Manterola D., C.; Bustos M., I. \& Vial G., M. Prevalencia de enfermedad por reflujo gastroesofágico en población general urbana adulta. Rev. Chil. Cir., 57(6):476-82, 2005.

Miner, P. Jr.; Katz, P. O. ; Chen, Y. \& Sostek, M. Gastric acid control with esomeprazole, lansoprazole, omeprazole, pantoprazole, and rabeprazole: a five-way crossover study. Am. J. Gastroenterol., 98(12):2616-20, 2003.

Nocon, M.; Labenz, J.; Jaspersen, D.; MeyerSabellek, W.; Stolte, M.; Lind, T.; Malfertheiner, P. \& Willich, S. N. Association of body mass index with heartburn, regurgitation and esophagitis: results of the Progression of Gastroesophageal Reflux Disease study. J. Gastroenterol. Hepatol, 22(11):1728-31, 2007.
Pessaux, P.; Arnaud, J. P.; Delattre, J. F.; Meyer, C.; Baulieux, J. \& Mosnier, H. Laparoscopic antireflux surgery: five-year results and beyond in 1340 patients. Arch. Surg., 140(10):946-51, 2005.

Salminen, P. T.; Hiekkanen, H. I.; Rantala, A. P. \& Ovaska, J. T. Comparison of long-term outcome of laparoscopic and conventional nissen fundoplication: a prospective randomized study with an 11-year follow-up. Ann. Surg., 246(2):201-6, 2007.

Scholten, T.; Teutsch, I. ; Bohuschke, M. \& Gatz, G. Pantoprazole on-demand effectively treats symptoms in patients with gastro-oesophageal reflux disease. Clin. Drug Investig., 27(4):287$96,2007$.

Sigterman, K. E.; van Pinxteren, B.; Bonis, P. A.; Lau, J. \& Numans, M. E. Short-term treatment with proton pump inhibitors, H2-receptor antagonists and prokinetics for gastrooesophageal reflux disease-like symptoms and endoscopy negative reflux disease. Cochrane Database Syst. Rev., 5:CD002095, 2013.

Vcev, A. ; Begic, I.; Ostojic, R. ; Jurcic, D. ; Bozic, D. ; Soldo, I.; Gmajnic, R.; Kondza, G.; Khaznadar, E. \& Micunovic, N. Esomeprazole versus pantoprazole for healing erosive oesophagitis. Coll. Antropol., 30(3):519-22, 2006.

Dirección para Correspondencia:

Prof. Dr. Carlos Manterola

Departamento de Cirugía

Universidad de La Frontera.

Casilla 54-D

Temuco

CHILE

Teléfono: 56-45-2325760

Fax: 56-45-2325761

Email: carlos.manterola@ufrontera.cl

Recibido : 06-11-2015

Aceptado: 14-01-2016 